\title{
Design of Smart Voice-Controlled Mini Car Based On SPCE061A
}

\author{
Hong-Jun GUa , Shi-Dong ZHU, Ding-Zhu XUE , Jian LI, Li-Xin HOU, \\ Shi-Jun LI ${ }^{\mathrm{b},}$ \\ ${ }^{1}$ Jilin Agricultural University, Changchun 130118, Jilin, China \\ aghj_dennis@163.com, bIsj0883@sina.com \\ *Shi-Jun LI
}

Keywords: voice control, mini car, SPCE061A, voice recognition.

\begin{abstract}
A smart voice-controlled mini car based on SPCE061A is designed. The single chip microcomputer (SCM) SPCE061A receives the analog voice signals collected by microphone, and converts them into digital signals through its inner $\mathrm{A} / \mathrm{D}$ converter. The digital signals can be compiled to some different commands, such as, 'forward', 'backward', 'left', 'right' and 'stop', which can control the actions of the car.
\end{abstract}

\section{Introduction}

In recent years, voice control system has been increasingly powerful with the development of electronic technology, and it has high requirements on reliability and accuracy[1]. Voice control has a broad prospect in application.

SCM has many advantages: small volume, low power consumption, strong control function, miniaturization, flexible extension, good integration and convenient use, etc[2]. And SCM can be used in intelligent instrument, household appliance, industrial control and agricultural field and so on. The SCM, SPCE061A from Sunplus Technology Co., Ltd is 16 bit microcontroller[3]. This SCM with the core of $\mu \mathrm{nSP}$ has the calculation function of DSP, the special function of which is voice recognition.[4]

Automobile industry has developed rapidly, and the price of cars is lower and lower. Cars has become the common transportation means for the people. Driverless car has entered the practical stage, so driving a car will not only rely on manual, but also voice control.

Voice control, as one of the auxiliary driving way of the future car, is worth research. This design centered on SPCE061A collects and deals with voice signals to form some commands. These commands can be matched with the commands prestored in a flash. So the research on SCM controlling a car (toy car) by voice signal recognition technology is practical significance[5].

\section{System Hardware Design}

\section{Overall Design Summary}

SPCE061A is adopted as the main controller in the system. Voice processing module integrated in the SCM can identify the voice signals accurately and effectively. SPC061A can control the rotation of the DC motors according to the input voice signals by small microphone. The audio output adopts a speaker with $8 \Omega$ and $0.5 \mathrm{~W}$. Infrared electric switch NPR220 is used to detect obstacles. And the system is supplied by four rechargeable batteries.

This design of hardware includes five modules: SPCE061A, power supply module, motor drive module, voice processing module and obstacle avoidable module.

Fig. 1 shows the car body structure view. The universal wheel in the front can walk all the directions. The rear wheels with the diameter $66 \mathrm{~mm}$ and made of black rubber tires are drived by DC geared motors. Turning and reversing of both rear wheels at the same time makes the car go forward and backward, respectively. Stopping left wheel and turning right wheel, or turning left wheel and 
stopping right wheel at the same time makes the car turn left or right. And when both wheels stop, the car stops. So the car has five motion states.

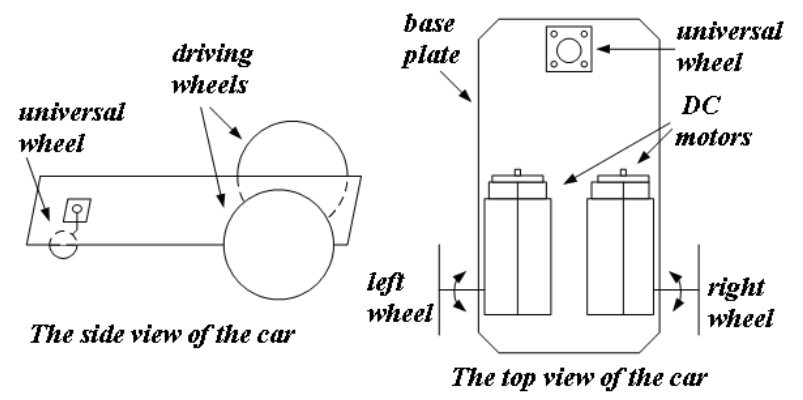

Fig. 1 The view of car body structure

All the modules are designed in the following.

\section{Voice recognition module}

Voice recognition has 'training' and 'recognizing' stages. In the training stage, SCM collects, analyzes and processes the voice sample. Voice feature information can be extracted to build a feature model. In the recognizing stage, SCM still extracts the voice feature information from voice signal and compare it to the feature model. When the comparison result reaches a certain compatibility, the voice signal is recognized. The principle is shown as Fig. 2.

\begin{tabular}{|c|c|c|c|}
\hline \multirow{2}{*}{ voice input $\sum$} & extract feature information & voice feature model & \\
\hline & training stage & & \\
\hline voice input $\sum$ & extract feature information & recognize & recognition result \\
\hline
\end{tabular}

Fig. 2 The voice recognition process

\section{Motor drive module}

As shown in Fig. 3, a DC motor drive circuit can be controlled by port A and port B. When both A and $B$ are input by ' 1 ', the motor stops. When $A$ and $B$ are input by ' 1 ' and ' 0 ', respectively, the current flows from M1 to M2, the motor can be regarded as 'turn'. When A and B are input by ' 0 ' and ' 1 ', respectively, the motor is in the 'reverse' state. It is noted that A and B must not be input ' 0 ' simultaneously, otherwise, the motor and transistors probably damage because of excessive current.

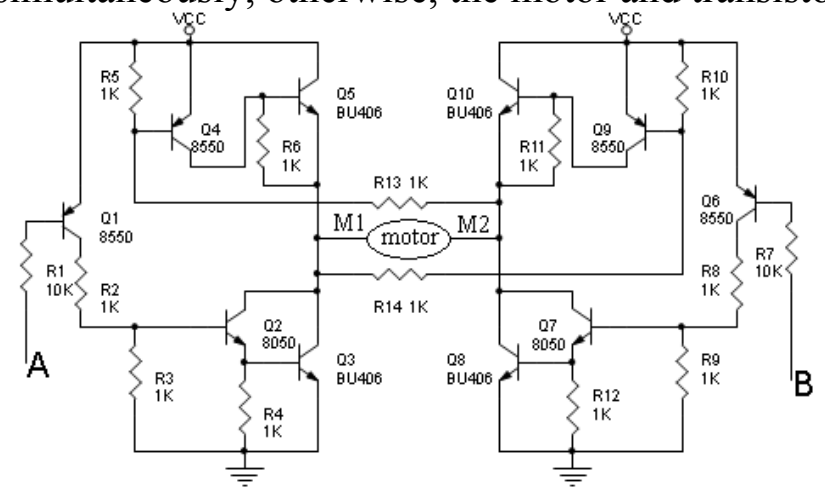

Fig. 3 The DC motor drive circuit

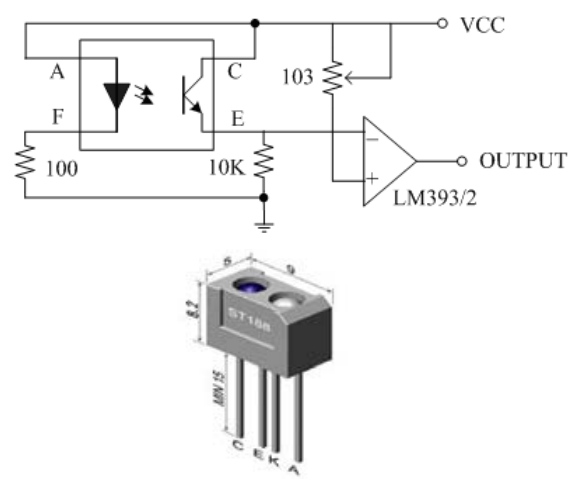

Fig. 4 The obstacle detection

\section{Obstacle detection module}

In Fig. 4, the obstacle detection circuit adopts the high power infrared transmitting and receiving tube ST188, the physical map of which is shown. When the sensor faces the obstacles (not too dark), the light which the transmission tube emits can be received by the receiving tube. Operational amplifier LM393 can convert the output signals into digital signals and send them to SCM. 


\section{System power supply module}

System power supply adopts four rechargeable batteries ( total voltage is about 6V ). SPEC061A needs $3.3 \mathrm{~V}$ power supply. The regulation chip SPY0029A can convert $6 \mathrm{~V}$ into $3.3 \mathrm{~V}$. The principle circuit is shown Fig. 5.

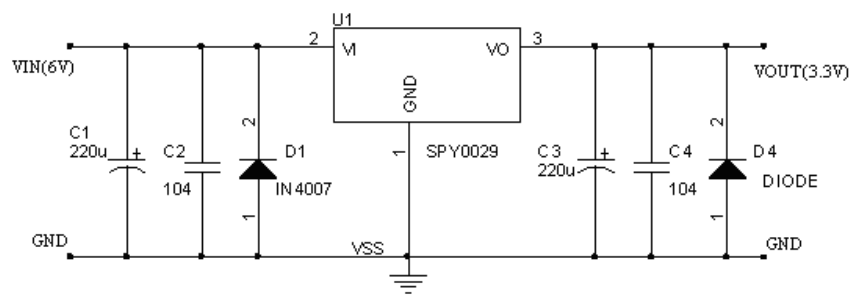

Fig. 5 The voltage conversion circuit

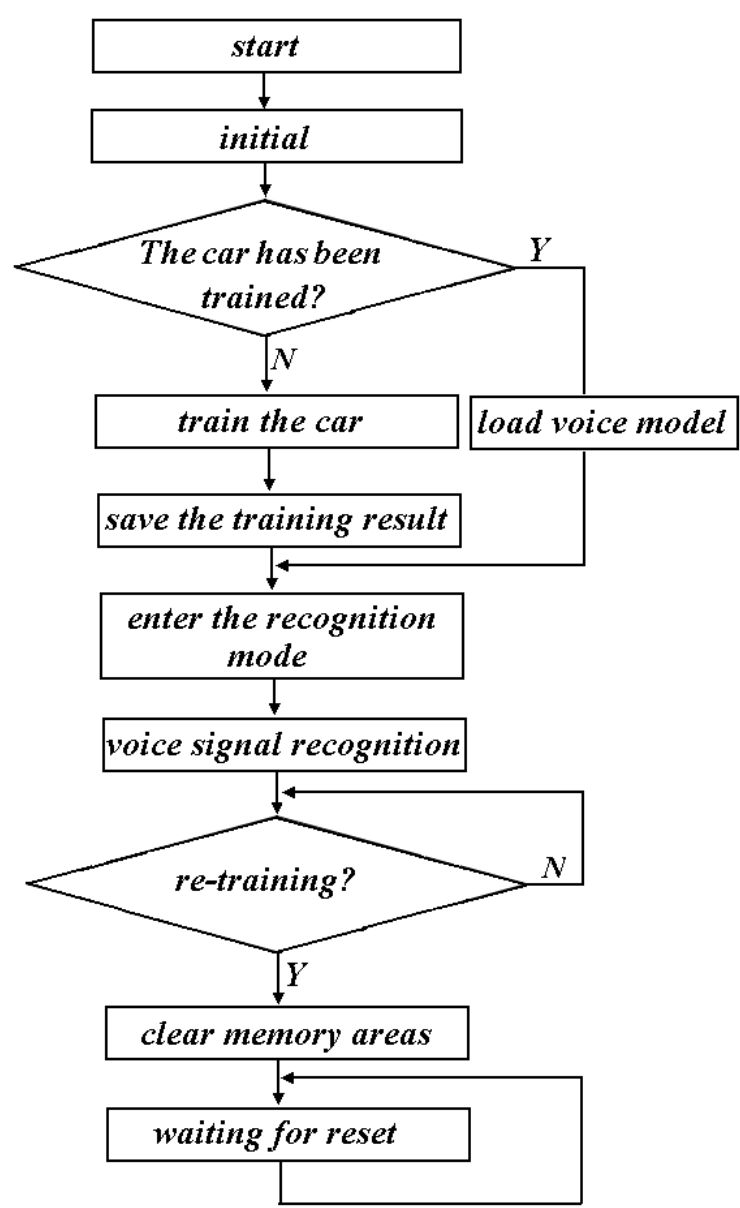

Fig. 6 The main program flow diagram

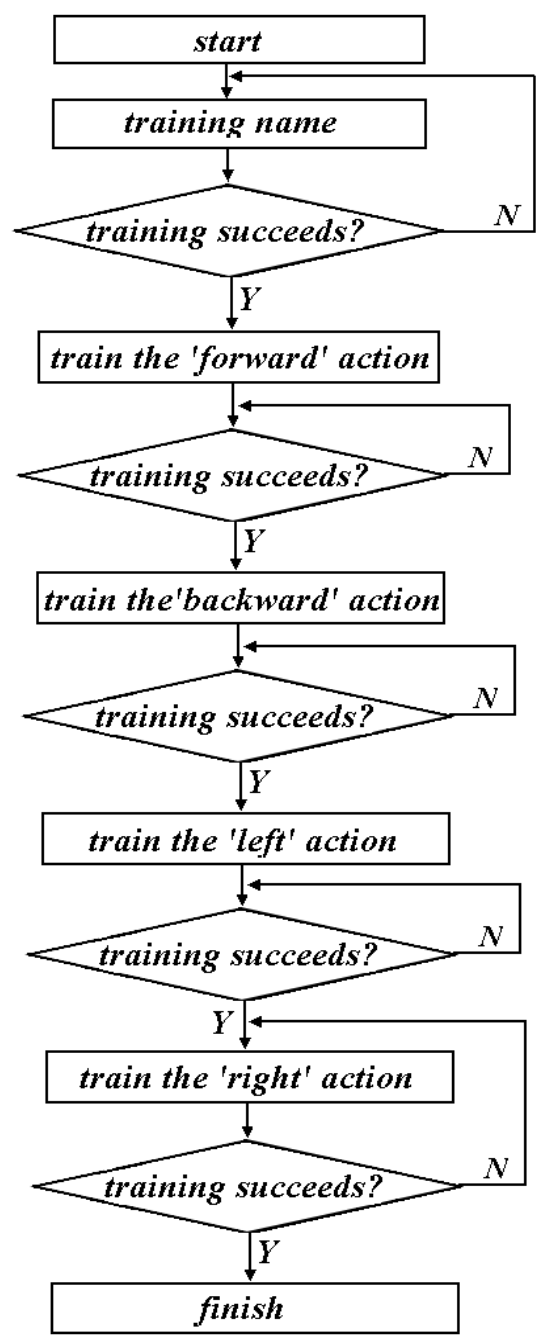

Fig. 7 The car training flow diagram

\section{System Software Design Flow Diagrams}

Fig. 6, Fig. 7 and Fig. 8 show the main program flow diagram, the car training subroutine flow diagram and the voice recognition subroutine flow diagram, respectively. Main program flow diagram includes initialization, training, recognition and re-training. In the initialization part, the most important job is that SCM assigns high levels to the ports controlling DC motor drivers, and initialize some variable and resisters. In the training part, voice model is built. If the car is not trained, it can get trained. After successful training, training model is stored in a flash, and no re-training when the training model is reused. If the car has been trained, the model in the flash is taken out to store in the recognizer. In the recognition part, the following gives the detailed executive process. In the re-training part, the system can do the re-training work when the corresponding key is pressed. 


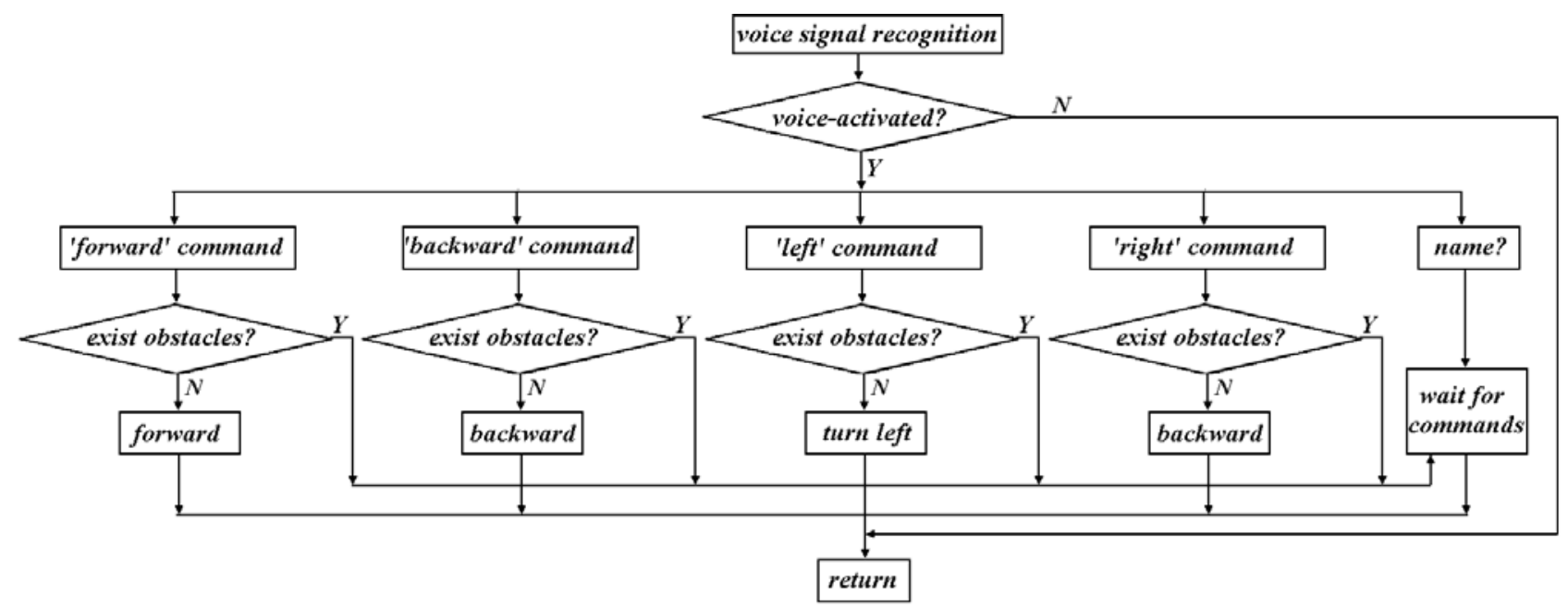

Fig. 8 The voice recognition flow diagram

As shown in Fig. 8, the car training subroutine adopts answering training. Voice commands can be input the system according to the sequence: the car's name, forward, backward, left and right.

As shown in Fig. 9, voice recognition subroutine firstly gains the recognition result and judge whether voice is activated. If voice is activated, ID number, representing the car's name or different action, returns. If ID represents a name, the car stops to wait the next voice signal. If ID represents a action, the car carries out the corresponding command.

\section{Conclusions}

This paper present a smart voice-controlled mini car based on SPCE061A. The car can carries out some commands, such as, 'name', 'forward', 'backward', 'left', and 'right'. Practice shows the car work stability and recognition success rate is high. As the driving auxiliary way, this system is profound significance to the driverless cars in the future.

\section{Acknowledgments}

The corresponding author, Shi-Jun LI, and other authors wish to express their gratitude to the projects, Jilin Province University Students' Innovation and Entrepreneurship Project, Jilin Province Economic Structural Adjustment Leading Fund Special Project (No. 2014Y108) and Changchun City Science and Technology Plan Project (No. 14nk029), Jilin Agricultural University, for their generous support of this work.

\section{References}

[1] Zhenyu Zhang, Hua Wang, Aihua Yu: Journal of Zhengjiang University of Science and Technology. Vol. 19(2007) p.27- 30, in Chinese.

[2] Shaocheng Qu, Ning Ai, Qiao Zhao:Electronic Measurement Technology. Vol. 31(2008) p.151154 , in Chinese.

[3] Dongbo Han, Bing Zeng, Liangquan Ge, et al: Computer Measurement \& Control. Vol. 15(2007) p.1183- 1185, in Chinese.

[4] Xiaoyong Shuai, Huanglin Tao, Jingxiang Lv: Journal of Jinggangshan University. Vol. 36 (2015) p.48-53, in Chinese.

[5] Xiaoyu Li, Yangming Lai, Shaohua Liu: Journal of Jiangxi University of Science and Technology. Vol. 32 (2011) p.53-55, in Chinese. 